\title{
Identification of Unknown Impurity of Azelaic Acid in Liposomal Formulation Assessed by HPLC-ELSD, GC-FID, and GC-MS
}

\author{
Stanisław Han, ${ }^{1}$ Katarzyna Karłowicz-Bodalska, ${ }^{1}$ Piotr Potaczek, ${ }^{2}$ Adam Wójcik, ${ }^{2}$ Lukasz Ozimek, ${ }^{2}$ \\ Dorota Szura, ${ }^{2}$ and Witold Musiat ${ }^{3,4,5}$
}

Received 10 March 2013; accepted 1 October 2013; published online 29 October 2013

\begin{abstract}
The identification of new contaminants is critical in the development of new medicinal products. Many impurities, such as pentanedioic acid, hexanedioic acid, heptanedioic acid, octanedioic acid, decanedioic acid, undecanedioic acid, dodecanedioic acid, tridecanedioic acid, and tetradecanedioic acid, have been identified in samples of azelaic acid. The aim of this study was to identify impurities observed during the stability tests of a new liposomal dosage form of azelaic acid that is composed of phosphatidylcholine and a mixture of ethyl alcohol and water, using high-performance liquid chromatography with evaporative light-scattering detector (HPLC-ELSD), gas chromatography-flame ionisation detection (GC-FID), and gas chromatography-mass spectrometry (GC-MS) methods. During the research and development of a new liposomal formulation of azelaic acid, we developed a method for determining the contamination of azelaic acid using HPLC-ELSD. During our analytical tests, we identified a previously unknown impurity of a liposomal preparation of azelaic acid that appeared in the liposomal formulation of azelaic acid during preliminary stability studies. The procedure led to the conclusion that the impurity was caused by the reaction of azelaic acid with one of the excipients that was applied in the product. The impurity was finally identified as an ethyl monoester of azelaic acid. The identification procedure of this compound was carried out in a series of experiments comparing the chromatograms that were obtained via the following chromatographic methods: HPLC-ELSD, GC-FID, and GC-MS. The final identification of the compound was carried out by GC with MS.
\end{abstract}

KEY WORDS: azelaic acid; GC-FID; GC-MS; HPLC-ELSD; impurities.

\section{INTRODUCTION}

Azelaic acid is a relatively new therapeutic substance that is used in the treatment of acne. Therapeutic treatment with $15 \%$ azelaic acid in a cream formulation has existed for two decades. Azelaic acid has several pharmacological effects: antibacterial, keratolytic, bleaching and metabolic (1). Its antibacterial activity is based primarily on its biocidal activity against Propioniobacterium acnes and Staphylococcus epidermidis. The drug also reduces the synthesis of keratin, leading to the suppression of comedogenic activity. Given its ability to inhibit the synthesis of melanin by inhibiting tyrosinase, azelaic acid is beneficial in the removal of melanin stains on the skin surface. Its inhibitory effect on 5-alpha-reductase

\footnotetext{
${ }^{1}$ Faculty of Pharmacy, Department of Industrial Pharmacy, Wrocław Medical University, Wrocław, Poland.

${ }^{2}$ Research and Development Center NOVASOME, Wrocław, Poland.

${ }^{3}$ Faculty of Pharmacy, Department of Pharmaceutical Technology, Wrocław Medical University, Borowska Str. 211A, 50-556, Wrocław, Poland.

${ }^{4}$ Present address: Faculty of Pharmacy, Department of Physical Chemistry, Wrocław Medical University, Borowska Str. 211A, 50556, Wrocław, Poland.

${ }^{5}$ To whom correspondence should be addressed. (e-mail: witold.musial@gmail.com)
}

reduces the formation of dihydrotestosterone that is normally synthesised in the skin from testosterone. Consequently, reduced lipogenesis in the affected tissue is observed $(2,3)$. To achieve an optimal therapeutic effect, several attempts have been made to modify the formulations of anti-acne drugs, including binding with ionic polymers (4) or evaluating the liposomal formulations (5).

Many impurities, such as pentanedioic acid, hexanedioic acid, heptanedioic acid, octanedioic acid, decanedioic acid, undecanedioic acid, dodecanedioic acid, tridecanedioic acid, and tetradecanedioic acid, have been identified in samples of azelaic acid. These impurities occur in acid azelaic as pharmaceutical raw material. They originate from contamination of the starting materials that are used for the preparation of azelaic acid by synthesis $(6,7)$ or by biotechnological methods (8). The contamination of pharmaceutical raw materials has been analysed during the research and development of active substances to prevent the side effects that are associated with their presence. Because of their toxic effects, the quantity of contaminants is often minimised in raw material $(9,10)$.

In the technology that is used to formulate specific dosages, a particularly relevant impact is the reaction of the excipients with the active agent that is present in the dosage form (11). Excipients, such as solvents, may interact with biologically active substances, resulting in the formation of pharmacologically inactive or toxic 
products (12). Many authors have studied the hydrolysis reactions that lead to decomposition of the active medicinal substance. One of the problems that have been observed in pharmaceutical compositions during stability tests is the esterification of the carboxyl groups in the presence of ethanol (13). In some cases, the interaction is beneficial $(14,15)$, but it typically leads to the formation of undesirable substances in the final product (16).

The identification of new contaminants is critical in the development of new medicinal products. The quantitative evaluation of dicarboxylic acids, including azelaic acid, may be performed using high-performance liquid chromatography with evaporative light-scattering detector (HPLC-ELSD) device (17) and many gas chromatography (GC) devices. GC, with the derivatisation of fatty acids to methyl esters, was used to determine the amount of azelaic acid in the oil paintings of old Flemish master painters (18). A similar method was applied for the evaluation of the composition of wall paintings (19). A modified GC method using a mass spectrometer detector (GC-MS) was applied to study the composition of Medieval and Renaissance Florentine paintings that originally contained proteins and fatty acids, including azelaic acid (20). GC-MS was also used to determine the content of azelaic acid in samples of air from the natural environment $(21,22)$. An interesting GC method to evaluate the content of azelaic acid in the natural aquatic environment was developed by Pusvaskiene et al. They used simultaneous derivatisation and dispersive microextraction using immiscible liquids, acetone, and carbon tetrachloride, whereas the ethylchloroformate was used as a reagent for derivatisation (23). The GC method was intensively developed due to the difficulties of determining the method's precision (24). The azelaic acid content in tobacco leaves was determined by the modified GC-MS method using methyl derivatives and by solid-phase microextraction (25). In a complex mixture of carboxylic, dicarboxylic, and amino acids, azelaic acid was assessed as a derivative of isobutylchloroformate using GC coupled with positive chemical ionization MS (GC-MS-PCI) (25).

The aim of this study was to identify impurities observed during the stability tests of a new liposomal dosage form of azelaic acid that is composed of phosphatidylcholine and a mixture of ethyl alcohol and water using HPLC-ELSD, gas chromatography-flame ionization detection (GC-FID), and GC-MS methods.

Table I. Composition of Mobile Phase During the Assay

\begin{tabular}{cccc}
\hline Time $[\mathrm{min}]$ & Phase A $(\%)$ & Phase B $(\%)$ & Phase C $(\%)$ \\
\hline 0 & 90 & 10 & 0 \\
3 & 90 & 10 & 0 \\
10 & 55 & 45 & 0 \\
40 & 15 & 85 & 0 \\
60 & 0 & 100 & 0 \\
63 & 0 & 100 & 0 \\
65 & 0 & 80 & 20 \\
71 & 0 & 80 & 20 \\
72 & 0 & 100 & 0 \\
76 & 0 & 100 & 0 \\
81 & 90 & 10 & 0 \\
88 & 90 & 10 & 0 \\
\hline
\end{tabular}

\section{MATERIALS AND METHODS}

HPLC-ELSD Agilent Technologies 1200; methanol (CHROMASOLV®, for HPLC, $\geq 99.9 \%$, Sigma-Aldrich); water (for HPLC, Sigma-Aldrich); chloroform (CHROMASOLV® Plus, for HPLC, $\geq 99.9 \%$, contains $0.5-1.0 \%$ ethanol as stabilizer, SigmaAldrich); $40 \%$ acetic acid (acetic acid solution for HPLC, SigmaAldrich); column: Merck, Purospher Star RP, C18, 250×4 mm, $(5 \mu \mathrm{m})$; reference sample of azelaic acid (AA; Fluka, serial number S38946); reference samples of identified impurities of azelaic acid, undecanedioic acid (I1, Aldrich, serial number: 1365985) and dodecanedioic acid (I2, Aldrich, serial number: MKBB0577); $0.20 \mu \mathrm{m}$ syringe filters; $2 \mathrm{~mL}$ syringes; HPLC probes. The analysis was performed in regard to the developed "in-house" new formulation of azelaic acid. The formulation composition included: azelaic acid, soya lecithin, ethyl alcohol, phosphate buffer $\mathrm{pH} 7.4$, and purified water.

\section{HPLC-ELSD}

For HPLC-ELSD analysis, we used a high-performance liquid chromatograph (Agilent Technologies 1200) and a Purospher Star RP, C18, $250 \times 4 \mathrm{~mm}(5 \mu \mathrm{m})$ column (Merck). Detection was performed by an ELSD detector. The following mixtures were used for the mobile phase: (A) methanol/water $(5: 95)+1.5 \%(v / v)$ acetic acid; (B) water/methanol $(5: 95)+1.5 \%(v / v)$ acetic acid; and $(C)$ chloroform. The analysis time was $88 \mathrm{~min}$; the gradient information is gathered in Table I.

Detection was performed using ELSD. The flow rate of the HPLC mobile phase was $1.0 \mathrm{~mL} / \mathrm{min}$, the column temperature was $30^{\circ} \mathrm{C}$, and the injection volume was $50 \mu \mathrm{L}$. The temperature of the nebulisation within the ELSD device was set at $40^{\circ} \mathrm{C}$ and the flow rate of the inert gas-nitrogen-inside the detector was set at $1.6 \mathrm{~mL} / \mathrm{min}$. The following samples were tested: a liposomal formulation of azelaic acid prepared without active substance (placebo, L), a liposomal formulation of azelaic acid with active substance (LA) and a reference sample of AA. The reference samples of the identified impurities (I1 and I2) were prepared by dissolving them in mobile phase B. During the evaluation of the assay method, the solutions of the individual impurities (I1 and I2) and a mixture of standards of known impurities (I1 and I2) were compared.

\section{GC-FID}

GC-FID was carried out using a gas chromatograph Agilent 7890 GC System. Two microlitres of the sample was injected onto the chromatographic column. Direct flow of the carrier gas (100:1 split) was provided with a flow rate of $0.8 \mathrm{~mL} / \mathrm{min}$. We used an HP-5MS column $(30 \mathrm{~m} \times 320 \mu \mathrm{m} \times 0.25 \mu \mathrm{m})$. The isothermal temperature of the chromatographic process was $70^{\circ} \mathrm{C}$, whereas the injector temperature was set at $270^{\circ} \mathrm{C}$ and the FID detector temperature was maintained at $300^{\circ} \mathrm{C}$. Helium carrier gas was applied in the GC column and the mixture of a combustible gas_-hydrogen $(35 \mathrm{~mL} / \mathrm{min})$ — and air $(350 \mathrm{~mL} / \mathrm{min})$ was used in the FID chamber. The active substance was processed as follows: $250 \mathrm{mg}$ of the active substance was mixed with $5 \mathrm{~mL}$ of a 2-M solution of chlorotrimethylsilane in methanol and then incubated for $16 \mathrm{~h}$ at $80^{\circ} \mathrm{C}$. The sample was filtered through a $0.45-\mu \mathrm{m}$ teflon syringe filter and injected after dilution with methanol for comparison with the reference solution. A sample of the final dosage form of the azelaic acid was prepared by dissolving $1.6 \mathrm{~g}$ of the 
Table II. Unidentified Impurity with a Retention Time of Approximately $31 \mathrm{Min}$ in the Liposomal Formulation of Azelaic Acid After 3 Months of Storage Under Various Conditions (Percent)

\begin{tabular}{lccc}
\hline $\begin{array}{l}\text { Test } \\
\text { conditions }\end{array}$ & $\begin{array}{l}\text { Temperature } \\
\left({ }^{\circ} \mathrm{C}\right)\end{array}$ & $\begin{array}{l}\text { Humidity } \\
(\% \mathrm{rH})\end{array}$ & $\begin{array}{l}\text { Concentration } \\
(\%)\end{array}$ \\
\hline Long-term & 25 & 60 & 0.11 \\
Intermediate & 30 & 60 & 0.76 \\
Accelerated aging & 40 & 75 & 3.33 \\
\hline
\end{tabular}

product in $10 \mathrm{~mL}$ of a 2-M solution of chlorotrimethylsilane in methanol and was then incubated for $16 \mathrm{~h}$ at $80^{\circ} \mathrm{C}$. The sample was filtered through a $0.45-\mu \mathrm{m}$ teflon syringe filter and injected into the analytical system without dilution.

\section{GC-MS}

The qualitative analysis of the unidentified impurity observed during the stability tests of liposomal formulation of azelaic acid was performed using an HP 5890 II gas chromatograph coupled with an HP 5971a mass selective detector. An HP-5MS capillary column (30 $\mathrm{m} \times$ $200 \mu \mathrm{m} \times 0.25 \mu \mathrm{m}$, film-cross-linked 5\% PH ME siloxane) was used with helium, as the carrier gas at a constant flow rate of $0.8 \mathrm{~mL} / \mathrm{min}$. The temperature of the column was programmed from $70^{\circ} \mathrm{C}$ to $290^{\circ} \mathrm{C}$ at $25^{\circ} \mathrm{C} / \mathrm{min}$, after an initial $1 \mathrm{~min}$ isothermal period and was maintained at the final temperature for $10 \mathrm{~min}$. The inlet was maintained at $290^{\circ} \mathrm{C}$. One microlitre of the sample was injected in the split mode (1:10) using a syringe. The mass spectrometer was set at an ionising voltage of $70 \mathrm{eV}$ with a mass range of $m / z$ 35-550. Sample preparation was carried out in the same manner as the GC-FID testing.

\section{RESULTS AND DISCUSSION}

During the research and development of a new liposomal formulation of azelaic acid (LA), we developed a method for determining the contamination of AA using HPLC with an ELSD detector. In preliminary studies of the stability of the new medicinal product, an unidentified impurity (NI) was observed with a retention time of approximately $31 \mathrm{~min}$. According to the results of the stability tests, the impurity appeared after three months of
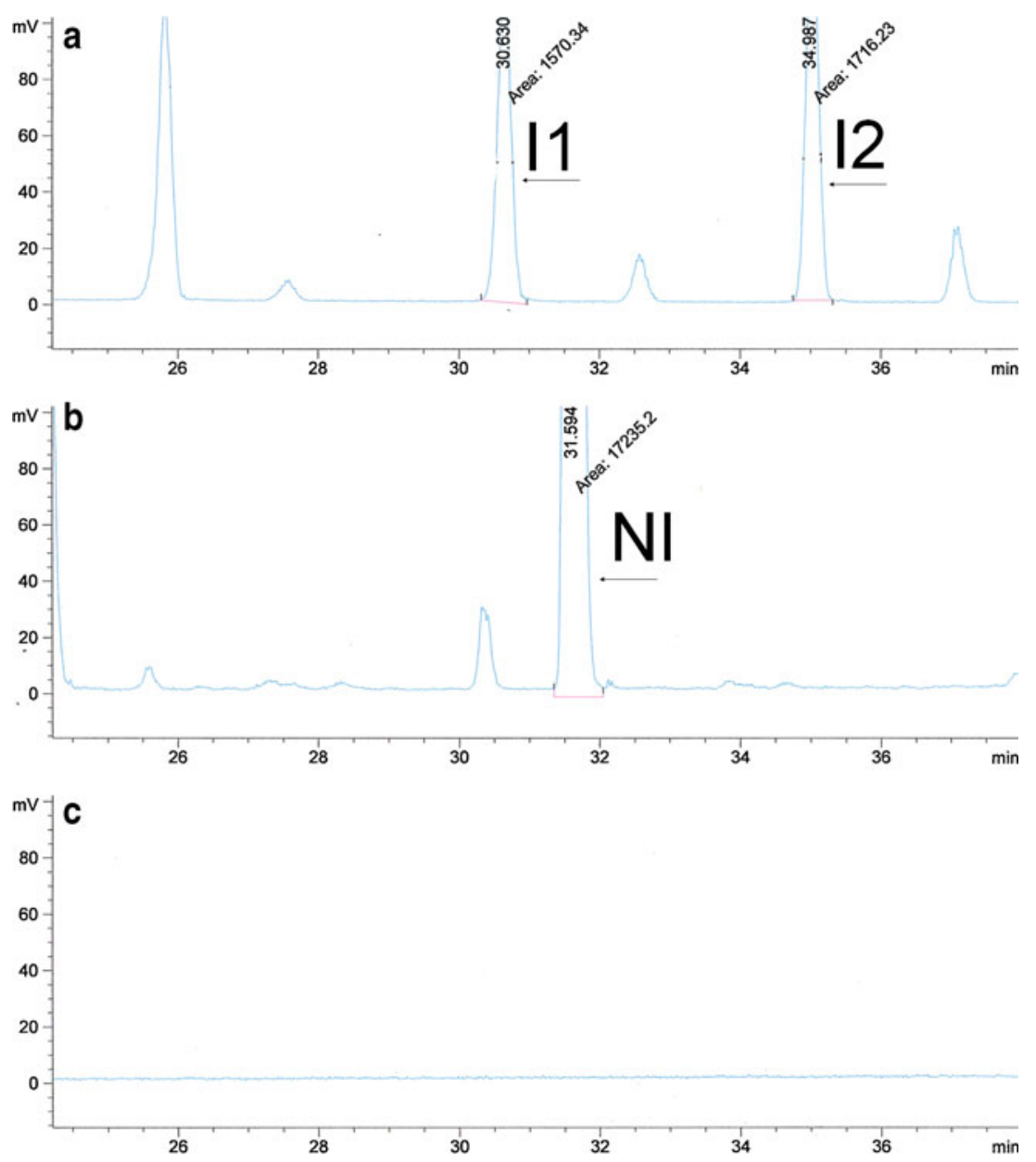

Fig. 1. Comparison of HPLC-ELSD chromatograms of a mixture of azelaic acid impurities I1 and I2 (a), liposomal formulation of azelaic acid LA (b), and samples not containing azelaic acid with a composition identical to the composition of the liposomal formulation $\mathrm{L}(\mathbf{c})$ 
testing the product in a long-term test, in an intermediate test and in accelerated aging tests, as shown in Table II. The abundance of the unidentified impurity NI with a retention time of approximately 31 min exceeded $0.1 \%$ of the calculated azelaic acid content. This $0.1 \%$ limit is consistent with the "in-house" specification that was evaluated according to the ICH standards (26).

\section{HPLC-ELSD Research}

During the tests using the product sample, the placebo sample, and the azelaic acid sample, under the standard stress conditions of high temperature and/or increased humidity, we showed that the peak with a retention time of approximately $31 \mathrm{~min}$ appeared only in the product sample (LA). The peak was not observed in the mixture of excipients referred to as placebo (L). This finding led to the conclusion that the unknown impurity is formed via the reaction of azelaic acid with one of the excipients of the liposomal formulation of azelaic acid.
The corresponding chromatograms are summarised in Fig. 1. Chromatogram 1A shows a mixture of two identified impurities (I1 and I2) that are similar to the unidentified impurities (NI). Chromatograms $1 \mathrm{~B}$ and 1C are from a liposomal formulation of azelaic acid with an active ingredient (LA) and the azelaic acid liposomal formulation without substance (L), respectively. The formulations were stored under stress conditions of elevated temperature and humidity, as indicated in Table I.

We followed several steps to identify the unknown impurity. In the first stage of the study, we performed the esterification reaction using AA and ethyl alcohol in the presence of sulphuric acid. Consequently, in the second stage we treated the liposomal formulation of azelaic acid (LA) with sulphuric acid. The obtained reaction mixture in the first case (AA) contained the ethyl monoester and ethyl diester of azelaic acid-Fig. 2a. The recorded retention times for respective peaks (Fig. 2a) enabled identification of $A A$ and NI in the mixtures presented on the Fig. $2 b, c$. In the second case, we obtained complex
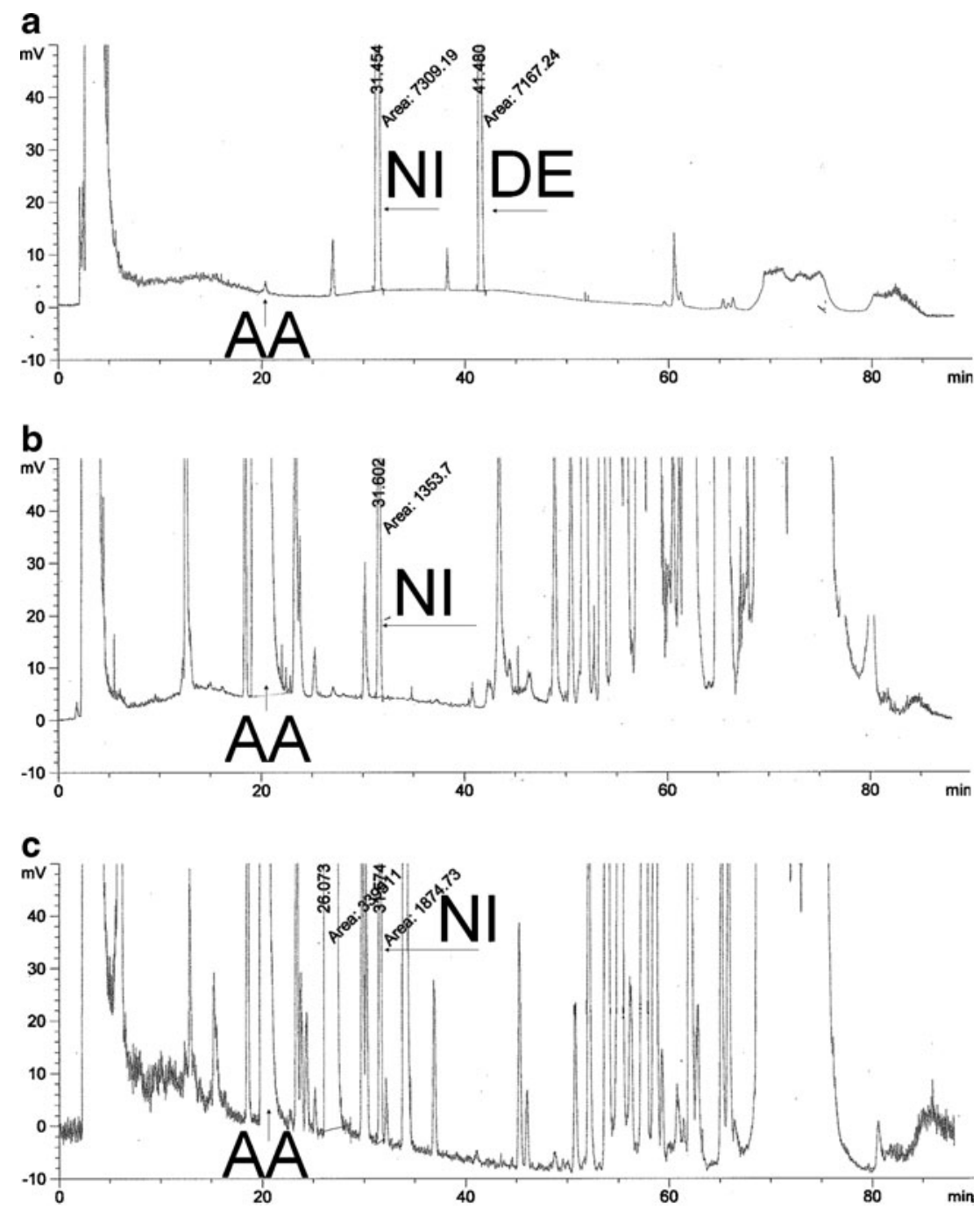

Fig. 2. HPLC-ELSD chromatograms of the reaction products of AA with ethanol in the presence of sulphuric acid $(A A-E t)(\mathbf{a})$, the liposomal formulation of azelaic acid stored for 12 months $(L A-A l t)(\mathbf{b})$, and the liposomal formulation of azelaic acid with ethanol in the presence of sulphuric acid $(L A-E t)(\mathbf{c})$ 
<smiles>CCOC(=O)CCCCCCCC(=O)O</smiles><smiles>CCOCCO</smiles><smiles>O=C(O)CCCCCCCC(=O)O</smiles><smiles>[2H]COCCO</smiles><smiles>CCOC(=O)CCCCCCCC(=O)OCC</smiles>

Fig. 3. Scheme of azelaic acid esterification with ethanol

mixtures, which were further evaluated. The mixtures were analysed using HPLC with an ELSD detector to determine the impurities of azelaic acid. The resulting chromatograms are shown in Fig. 2, along with a chromatogram of a sample of liposomal formulation of azelaic acid that was stored for 12 months in ambient conditions.

There are two main peaks in the chromatogram of the sample reaction mixture of AA with ethyl alcohol in the presence of sulphuric acid (Fig. 2a) that correspond to the retention times of the peaks that were observed in the chromatogram of the test sample of the liposomal formulation of azelaic acid stored through 12 months (Fig. 2b). Similar maxima were observed in the case of samples of AA liposomal formulation after addition of sulphuric acid as an esterification accelerator (Fig. 2c). These peaks are the AA peak, with a retention time of approximately $20.5 \mathrm{~min}$ and the unidentified impurity (NI) peak, with a retention time of approximately $31.5 \mathrm{~min}$. Additionally, we observed another peak at ca. $41.5 \mathrm{~min}$ in the chromatogram of the reaction mixture; we attributed it to the diethyl derivative of AA. It was not present in the liposomal formulation, due to the low concentration of ethyl alcohol used in the production process, compared with reaction mixture AA with ethyl alcohol. Based on that observation, the unidentified impurity of azelaic acid could be produced by the esterification reaction and could be defined as a monoethyl ester of azelaic acid, as shown in Fig. 3.

\section{GC-FID Studies}

In the next stage of the analysis, we performed method optimisation for the detection of AA of pollutants using GC-FID. Prior to being applied to the column, the samples were derivatised with a silylating reagent, hexamethyldisilazane, according to the reaction scheme shown in Fig. 4.

The chromatograms that were acquired during the analysis of the following samples in the GC-FID device are presented in Fig. 5: silylated sample of the reaction mixture with ethanol and azelaic acid, designated AA-EtSil (Fig. 5a); silylated products of the liposomal formulation of azelaic acid prepared "ex tempore," designated LA-Sil-1 (Fig. 5b); and silylated products of the liposomal formulation of azelaic acid stored for 12 months, designated LA-Sil-2 (Fig. 5c). The chromatogram of the AAEt-Sil (Fig. 5a) contains two peaks corresponding to the retention times of the peaks that are observed in the chromatogram of LA-Sil-2 (Fig. 5c), which is the test sample of the liposomal formulation of azelaic acid that was stored for 12 months. The retention time of $16.9 \mathrm{~min}$ corresponds to AA, whereas the retention time of approximately $16.6 \mathrm{~min}$ corresponds to the unidentified impurity (NI). In the sample prepared ex tempore (Fig. 5b), we did not observe the characteristic peak at 16.6 for NI. Both in<smiles>O=C(O)CCCCCCCC(=O)O</smiles>

$\mathrm{AA}$
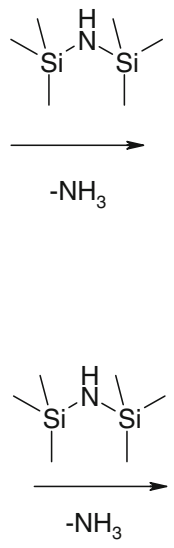

AA<smiles>C[Si](C)(C)OC(=O)CCCCCCC(=O)O[Si](C)(C)C</smiles>

AA-Sil

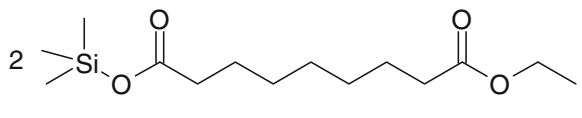

ME-AA-Sil

Fig. 4. Scheme of the reaction of the silylation of azelaic acid (first row) and azelaic acid ethyl ester (second row) 

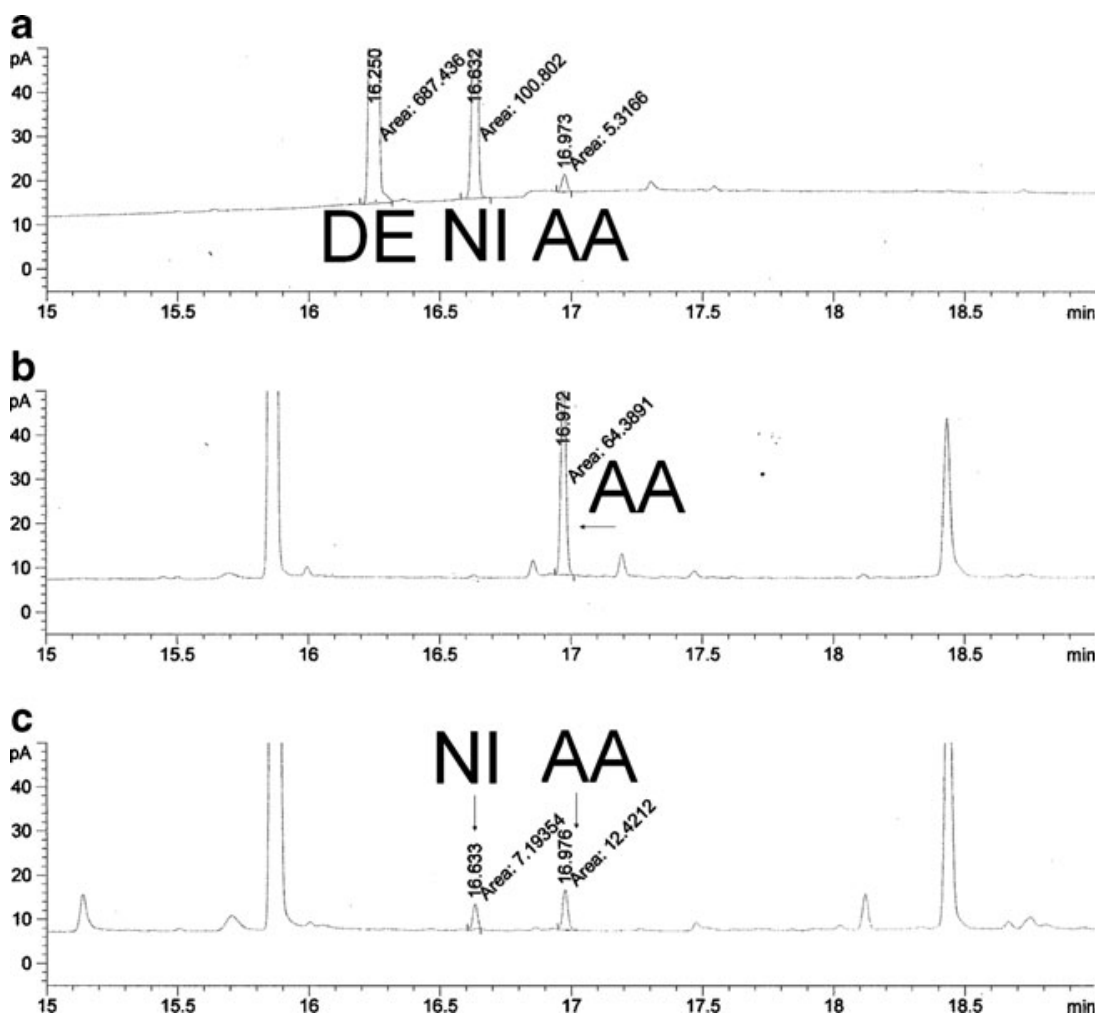

Fig. 5. GC-FID chromatograms: a silylated sample of the reaction mixture with ethanol and azelaic acid $(A A-E t-S i l)$; b silylated products of the liposomal formulation of azelaic acid prepared "ex tempore" ( $L A$-Sil-1); and $\mathbf{c}$ silylated products of the liposomal formulation of azelaic acid stored for 12 months $(L A-S i l-2)$

the case of LA-Sil-1 and LA-Sil-2, the maxima observed at $15.9 \mathrm{~min}$ and at around $18.4 \mathrm{~min}$ come from the components of liposomal formulation (Fig. 5b, c)—we did not observe them in the chromatogram of reaction mixture of AA and ethyl alcohol (Fig. 5a).

During the analysis performed using HPLC-ELSD and GC-FID measurement, we attempted to elucidate the nature of the unidentified impurity by comparing the position of the peak that was observed in the measurement of the liposomal formulation of azelaic acid and the peak of azelaic acid that was present in the reaction mixture that was treated with ethyl alcohol in the presence of sulphuric acid.

\section{GC-MS Studies}

The third step of the identification of the compound was performed by comparing the mass spectra obtained during analysis of the samples, using a GC-MS device, after derivatisation with a silylating reagent, as mentioned above. The analysed samples comprised a mixture of ethyl esters of azelaic acid, named AA-Et-Sil and samples of the two series of liposomal formulations of azelaic acid: one batch produced ex tempore, named LA-Sil-1 and one batch that was stored for 12 months in ambient conditions, named LA-Sil-2. As shown in Fig. 6, there are three peaks in the chromatogram of the silylated mixture of reacted azelaic acid (AA-Et-Sil): a peak with a retention time of $7.77 \mathrm{~min}$ that was derived from the ethyl diester of azelaic acid (DE-AA), a peak at a retention time of $8.03 \mathrm{~min}$ that was derived from the silylated ethyl monoester of azelaic acid (ME-AA-Sil), and a peak with a retention time of 8.28 min that was derived from silylated azelaic acid (AA-Sil). Also, shown in Fig. 6 are the mass spectra of the individual peaks.

The silylated peak of azelaic acid was identified by comparison with known mass spectra that were obtained from an available database. Confirmation of the position of the peak identified as the silylated monoester of azelaic acid was based on the work of Van der Berg, who studied the mass spectra of dicarboxylic acids and showed that the mass spectrum of the GC-MS peak with a retention time of $8.03 \mathrm{~min}$ corresponds to the derivative of the silylated ethyl monoester of azelaic acid. Comparison of the two spectra confirms that the peak obtained experimentally in our work is a silylated monoester derivative of azelaic acid. Additionally, comparison of the mass spectra of the peak with a retention time of 8.0 min that is derived from a mixture of esters of azelaic acid (AA-Et-Sil) and the test sample of the liposomal formulation of azelaic acid that was stored for 12 months (LA-Et-Sil-2) confirms that the two peaks are derived from the same compound, in accordance with the data shown in Fig. 7.

Additional verification of the chromatograms and confirmation of accurate identification of the assessed impurity as the ethyl monoester of azelaic acid was carried out by comparing the GC-MS chromatograms of the following samples: the liposomal formulation of azelaic acid 


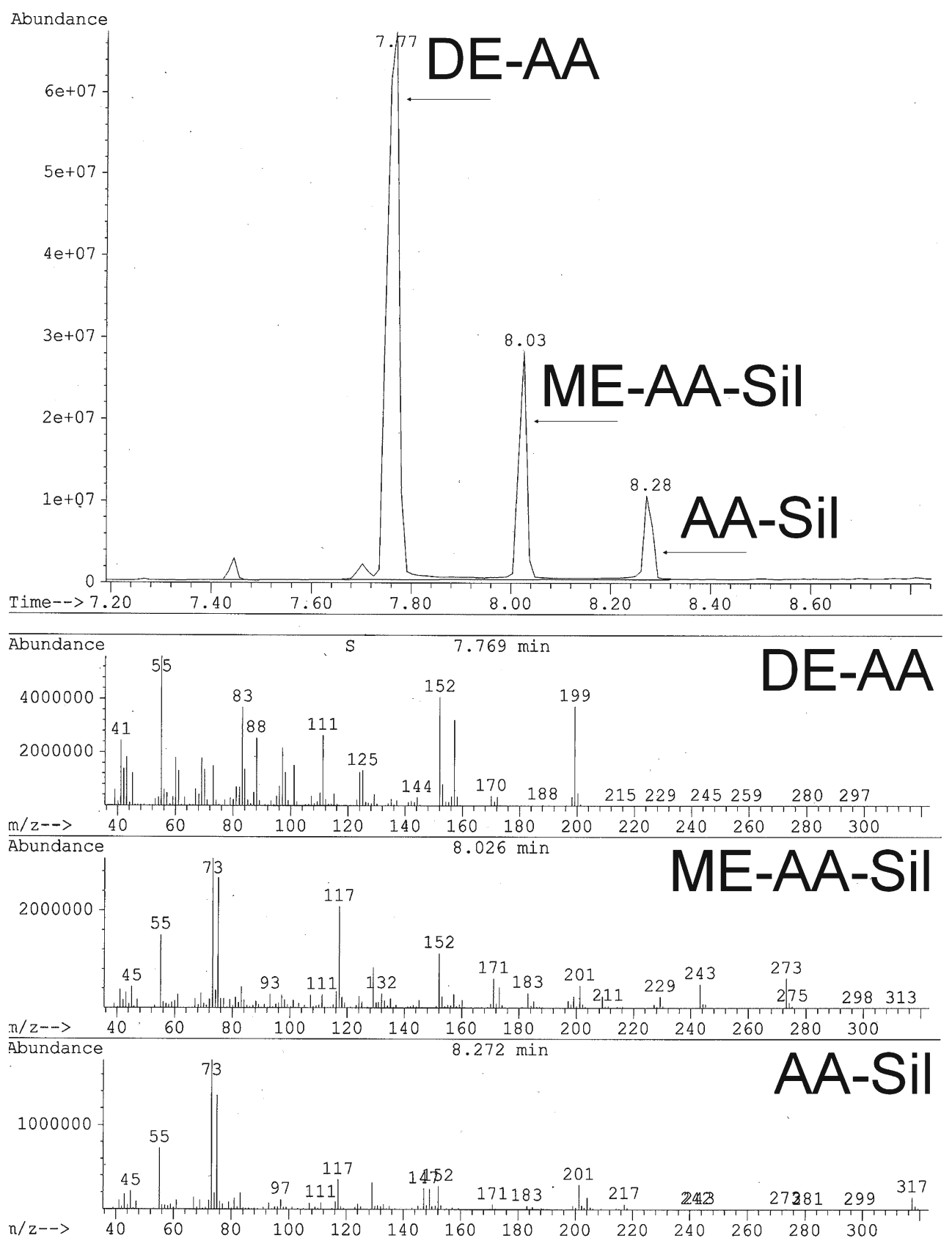

Fig. 6. GC chromatograms obtained from the measurements of the silylated products of the reaction of azelaic acid with ethanol in the presence of sulphuric acid $(A A-E t-S i l)$ with the corresponding mass spectra

prepared ex tempore (LA-Et-Sil-1) and the liposomal formulation of azelaic acid that was stored for 12 months (LA-Et-Sil-2), as shown in Fig. 8. The chromatogram of the tested sample of the liposomal formulation of azelaic acid that was stored for 12 months shows a distinct peak originating from the ethyl monoester of azelaic acid, with a retention time of approximately $8.00 \mathrm{~min}$. A similar but less distinct peak is observed in the chromatogram of the sample of the liposomal formulation of azelaic acid, prepared ex tempore; the visible small peak was derived from the ethyl monoester of azelaic acid and has a retention time of $8.02 \mathrm{~min}$. The peak at ca. $7.77 \mathrm{~min}$ may be attributed to the diethyl derivative of azelaic acid, however detailed analysis needs further research, with extended evaluation of GC-MS spectra.

During the assessments, we observed some peaks in the HPLC-ELSD chromatograms which may be attributed to the diethyl ester of AA. Actually, in the reaction of AA with ethanol, there should be two esters formed: ethyl ester and diethyl ester of AA, as it is presented on the Fig. 3. We identify the peak at $41.4 \mathrm{~min}$ as the possible diethyl ester of AA-Fig. 2a (DE); however, the investigation of this derivative of AA will be performed in another study. The applied silylation reaction, which enables derivatization, occurs in the presence of available hydroxyl groups, which are blocked in the dietyl ester of AA, so further research will be planned. 


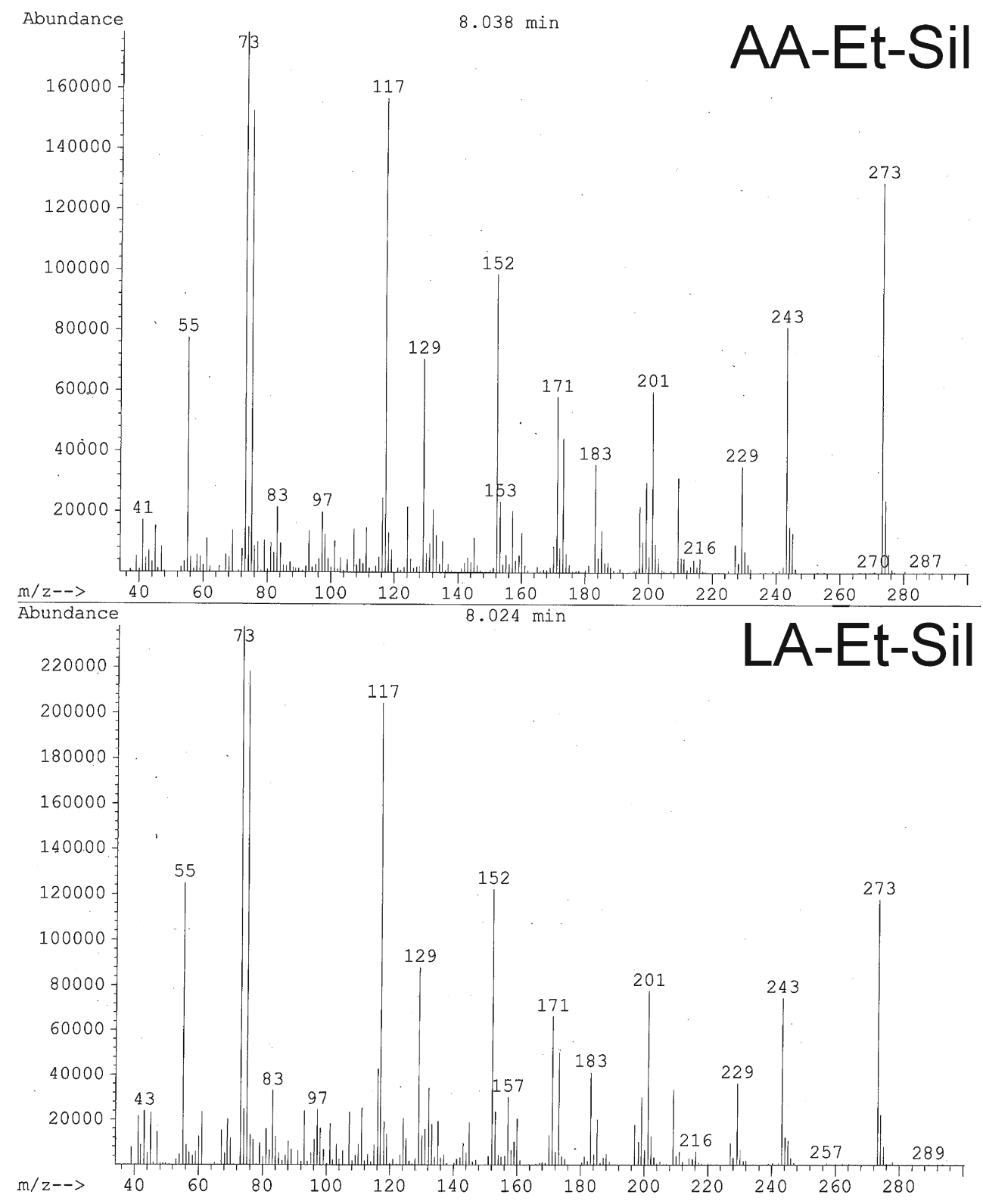

Fig. 7. Mass spectra of two peaks with a retention time of approximately $8.0 \mathrm{~min}$ : AA-Et-Sil mass spectrum of the silylated derivative of the monoester of azelaic acid that was obtained on the silylated products of the reaction of azelaic acid with ethanol in the presence of sulphuric acid; $L A$-Et-Sil-2 silylated products of the reaction of the liposomal formulation of azelaic acid with ethanol in the presence of sulphuric acid after 12 months of storage

\section{CONCLUSIONS}

During our analytical tests, we identified a previously unknown impurity of a liposomal preparation of azelaic acid that appeared in the liposomal formulation of azelaic acid during preliminary stability studies. The results led to the conclusion that the impurity was caused by the reaction of azelaic acid with one of the excipients that was applied in the product, i.e., with ethanol. The impurity was finally identified as an ethyl monoester of azelaic acid. The identification procedure of this compound was carried out in a series of experiments comparing the chromatograms that were obtained via the following chromatographic methods: HPLC-ELSD, GC-FID, and GC-MS. In our study, we synthesized an in-house ethyl monoester of azelaic acid by performing the esterification reaction of azelaic acid. The similarity of the retention time of the obtained compound to that of an unidentified peak in the chromatogram of the sample of the liposomal formulation of azelaic acid was confirmed by two independent methods of chromatographic separation: HPLC-ELSD and GCFID. The final identification of the compound was carried out by GC with MS. The final product and the mixture of esters had identical mass spectra. Furthermore, the mass 


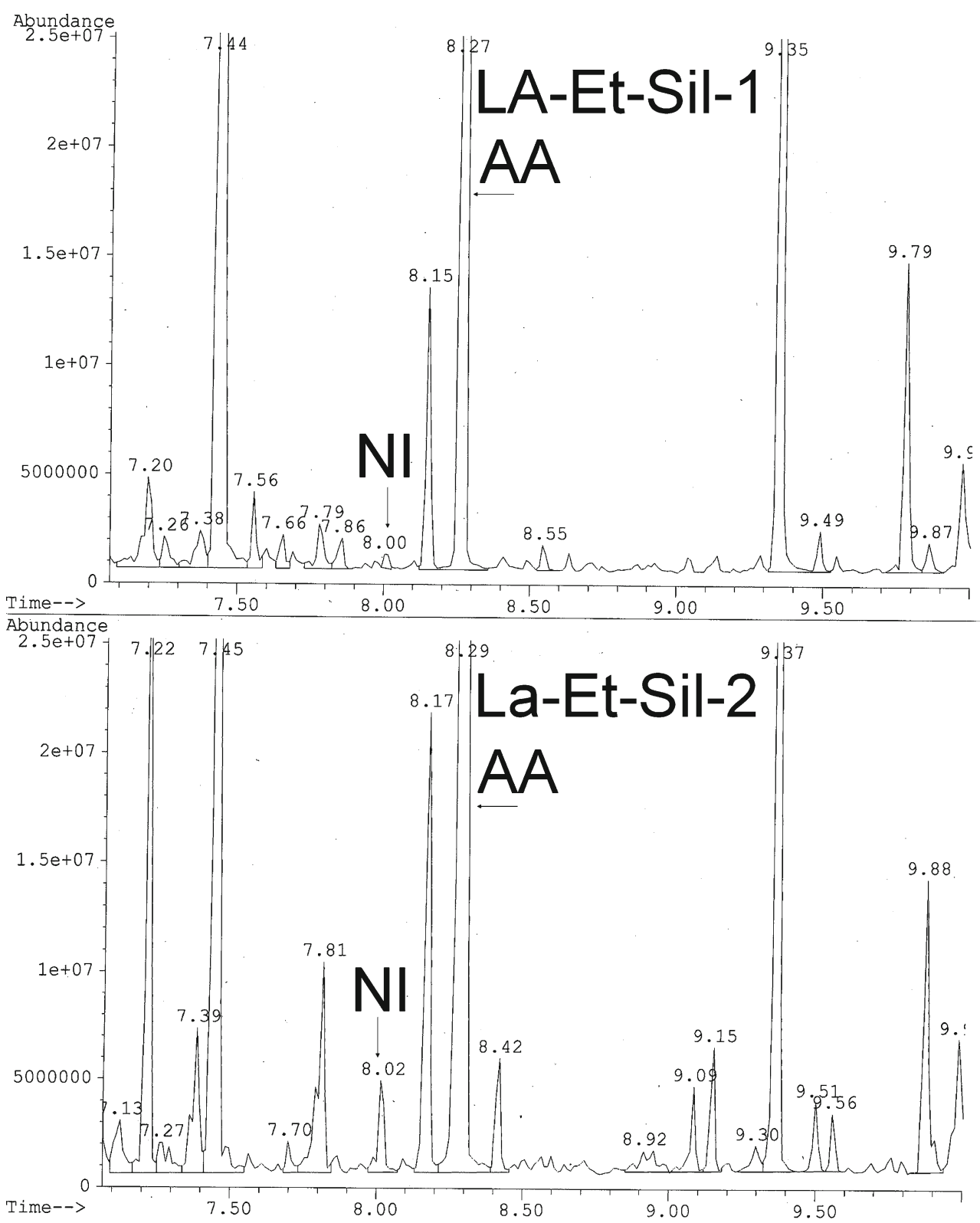

Fig. 8. GC-MS chromatograms obtained from the silylated products of the reaction of azelaic acid in the liposomal formulation "ex tempore" (LA-Et-Sil-1) and stored for 12 months (LA-Et-Sil-2) and the corresponding peaks of azelaic acid $(A A)$ and the unidentified impurity $(N I)$

spectrum present in the literature confirms that the generated impurity of azelaic acid is the ethyl monoester of azelaic acid. Some of the peaks within the analyzed spectra suggest the presence of diethyl ester of azelaic acid; however, we did not observe diethyl ester of azelaic acid within the liposomal preparations.

\section{ACKNOWLEDGMENTS}

This publication was co-financed by the Wrocław Research Center EIT + under the auspices of the European Social Fund, project no POKL.08.02.01-02-011/11. The GC-MS measurements were performed with the kind assistance of Marek Hojniak from the Faculty of Chemistry, University of Wrocław.
Open Access This article is distributed under the terms of the Creative Commons Attribution License which permits any use, distribution, and reproduction in any medium, provided the original author(s) and the source are credited.

\section{REFERENCES}

1. Bladon PT, Burke BM, Cunliffe WJ, Forster RA, Holland KT, King K. Topical azelaic acid and the treatment of acne: a clinical and laboratory comparison with oral tetracycline. Br J Dermatol. 1986;114(4):493-9.

2. Stamatiadis D, Bulteau-Portois MC, Mowszowicz I. Inhibition of 5 alpha-reductase activity in human skin by zinc and azelaic acid. Br J Dermatol. 1988;119(5):627-32. 
3. Thiboutot D, Harris G, Iles V, Cimis G, Gilliland K, Hagari S. Activity of the type 15 alpha-reductase exhibits regional differences in isolated sebaceous glands and whole skin. J Invest Dermatol. 1995;105(2):20914.

4. Musial W, Kubis A. Preliminary assessment of alginic acid as a factor buffering triethanolamine interacting with artificial skin sebum. Eur J Pharm Biopharm. 2003;55(2):237-40.

5. Esposito E, Menegatti E, Cortesi R. Ethosomes and liposomes as topical vehicles for azelaic acid: a preformulation study. J Cosmet Sci. 2004;55(3):253-64.

6. Goebel CG, Brown AC, Oehlschlaeger HF, Rolfes RP. Method of Making Azelaic Acid. United States Patent No. 2,813,113. 1957.

7. Frische R, Hegwein K, Volkheimer J. Method of producing dicarboxylic acids suitable for synthesis of polymers or polyamides. United States Patent No. 6,362,368. 2002.

8. Nghiem NP, Donnelly M, Millard CS, Stols L. Method for the production of dicarboxylic acids. US Patent No. 5,869,301. 1999.

9. Jacobson-Kram D, McGovern T. Toxicological overview of impurities in pharmaceutical products. Adv Drug Deliv Rev. 2007:59(1):38-42.

10. Muller L, Mauthe RJ, Riley CM, Andino MM, Antonis DD, Beels C, et al. A rationale for determining, testing andcontrolling specific impurities in pharmaceuticals that possess potential for genotoxicity. Regul Toxicol Pharmacol. 2006;44(3):198-211.

11. Bharate SS, Bharate SB, Bajaj AN. Incompatibilities of pharmaceutical excipients with active pharmaceutical ingredients: a comprehensive review. J Excip Food Chem. 2010;1(3):3-26.

12. Arakawa T, Kita Y, Carpenter JF. Protein-solvent interactions in pharmaceutical formulations. Pharm Res. 1991;8(3):285-91.

13. Irwin WJ, Masuda QN, Li Wan Po A. Transesterification: an analytical and formulation problem. J Pharm Biomed Anal. 1985;3(3):241-50.

14. Alander JT. Chemical and physical properties of emollients. Treat Dry Skin Synd. 2012;399-417.

15. Kalinkova G. Studies of beneficial interactions between active medicaments and excipients in pharmaceutical formulations. Int $\mathrm{J}$ Pharm. 1999;187(1):1-15.

16. Foresti ML, Galle M, Ferreira ML, Briand LE. Enantioselective esterification of ibuprofen with ethanol as reactant and solvent catalyzed by immobilized lipase: experimental and molecular modeling aspects. J Chem Technol Biotechnol. 2009;84(10):1461-73.

17. Bravi E, Perretti G, Montanari L. Fatty acids by high-performance liquid chromatography and evaporative light-scattering detector. J Chromatogr A. 2006;1134(1):210-4.

18. Schilling MR, Khanjian HP, editors. Gas chromatographic determination of the fatty acid and glycerol content of lipids: I. The effects of pigments and aging on the composition of oil paints. ICOM committee for conservation, 11th triennial meeting in Edinburgh, Scotland, 1-6 September 1996: Preprints; 1996: James \& James (Science Publishers) Ltd.

19. Colombini MP, Modugno F, Giacomelli M, Francesconi S. Characterisation of proteinaceous binders and drying oils in wall painting samples by gas chromatography-mass spectrometry. J Chromatogr A. 1999;846(1):113-24.

20. Colombini MP, Modugno F, Menicagli E, Fuoco R, Giacomelli A. GC-MS characterization of proteinaceous and lipid binders in UV aged polychrome artifacts. Microchem J. 2000;67(1):291-300.

21. Yokouchi Y, Ambe Y. Characterization of polar organics in airborne particulate matter. Atmos Environ. 1967;20(9):1727-34.

22. Kawamura K, Watanabe T. Determination of stable carbon isotopic compositions of low molecular weight dicarboxylic acids and ketocarboxylic acids in atmospheric aerosol and snow samples. Anal Chem. 2004;76(19):5762-8.

23. Pusvaskiene E, Januskevic B, Prichodko A, Vickackaite V. Simultaneous Derivatization and dispersive liquid-liquid microextraction for fatty acid GC determination in water. Chromatographia. 2009;69(3):271-6.

24. Cappa CD, Lovejoy ER, Ravishankara A. Determination of evaporation rates and vapor pressures of very low volatility compounds: a study of the C4-C10 and C12 dicarboxylic acids. J Phys Chem A. 2007;111(16):3099-109.

25. Clark TJ, Bunch JE. Derivatization solid-phase microextraction gas chromatographic-mass spectrometric determination of organic acids in tobacco. J Chromatogr Sci. 1997;35(5):209-12.

26. ICH Harmonised Tripartite Guideline. Validation of analytical procedures: Text and methodology Q2 (R1). Geneva: IFPMA; 2005. 